\title{
Numerical Advances in the Full-Wave Analysis of Periodic Multilayered Structures with Application to Reflectarray Antennas
}

\author{
Rafael Florencio, Rafael Rodríguez Boix, and José A. Encinar
}

\begin{abstract}
A novel hybrid spectral-spatial method of moments (MoM) approach is introduced for the analysis of multilayered periodic structures containing either patches or dipoles in the unit cell. The novel hybrid MoM approach and the pure spectral MoM approach are both applied to the analysis and design of reflectarray antennas made of patches or dipoles under the local periodicity assumption. The results indicate that the hybrid approach is always at least one order of magnitud faster than the spectral approach in the analysis and design of the antennas.
\end{abstract}

\section{INTRODUCTION}

Reflectarray antennas are an interesting alternative to reflector antennas and phased arrays. The main drawback of reflectarray antennas is their narrow bandwidth [1]. Fortunately, the bandwidth problem has been solved to a large extent by the introduction of multi-resonant elements, and by the use of optimization techniques that enforce the phase requirements in each reflectarray element at several frequencies. The combined use of these two strategies has made it possible to design contoured beam dual-frequency dualpolarization reflectarray antennas with stringent requirements in which the elements are either three stacked rectangular patches [2] or two orthogonal sets of parallel dipoles [3], [4]. The design of reflectarray antennas is customarily based on the local periodicity assumption, which implies that the dimensions of each element that lead to the required phase shift (for a prescribed radiation pattern) are adjusted as if the element were placed in a periodic environment. This means that the design of a large reflectarray antenna (containing several thousands elements) optimized in one or two frequency bands requires the numerical analysis of a huge number of multilayered periodic structures, and therefore, very efficient numerical tools are required for the analysis of those periodic structures. The numerical technique traditionally employed in the analysis of multilayered periodic structures is the method of moments (MoM) in the spectral domain [5]. Unfortunately, the direct application of this technique is not sufficiently fast because it requires the lengthy computation of slowly convergent double infinite summations for the determination of the MoM matrix entries involving basis functions (BFs) for the current density at the same metallization level. In this paper, we introduce a novel hybrid spectral-spatial MoM approach for the analysis of multilayered periodic structures containing either patches or dipoles, which is specifically devised for reflectarray design. In the novel approach the MoM matrix entries involving $\mathrm{BF}$ at different metallizations levels are computed in the spectral domain as double infinite summations with fast exponential convergence. However, the MoM matrix entries involving $\mathrm{BF}$ at the same metallization level are computed in the spatial domain as double finite integrals which can be efficiently computed by using low order quadrature rules. Numerical simulations have shown that the new hybrid MoM is between 10 and 30 times faster than the traditional spectral MoM in the design of reflectarray antennas made of stacked rectangular patches [6], and in the design of reflectarray antennas made of orthogonal sets of parallel dipoles.

\section{BRIEF OUTLINE OF THE NUMERICAL PROCEDURE}

As commented in the introduction, in this work the MoM matrix entries involving $\mathrm{BF}$ at the same metallization level have been efficiently computed in the spatial domain as double finite integrals. The integrands of these integrals are multilayered periodic Green's functions (MPGFs) times crosscorrelations between the BF and their divergences. The MPGF are expressed as double infinite summations, and the computation of these summations is substantially accelerated by the combined use of Kummer's transformation, the discrete complex image method for the determination of the asymptotic terms, and Ewald's method [6]. To further minimize the total number of MPGF that have to be computed, Chebyshev polynomials have been used to interpolate in four dimensions these MPGF in terms of the integration variables of the MoM matrix entries [6], and in terms of the angles of incidence of the wave impinging on the periodic structures. To avoid singularity interference in the interpolation process, prior to interpolation, we have extracted in closed-form the source singularity and the images of the source through the closest layers of the multilayered substrate [6]. Also, we have extracted in closed- 
form the spectral terms of the MPGF containing the poles introduced by multilayered surface waves. Chebyshev polynomials weighted by edge condition functions have been used as $\mathrm{BF}$ in the approximation of the current density on both patches and dipoles since these $\mathrm{BF}$ ensure a fast convergence of MoM with respect to the number of $\mathrm{BF}$ [7]. The cross-correlations between these $\mathrm{BF}$ and their divergences have been obtained in closed-form in terms of complete and normal elliptic integrals of the first and second kind for which efficient algorithms exist [8]. Since the singularities of the integrands have a deleterious effect on the numerical integration procedure, on the one hand the source singularities of the MPGF have been extracted and integrated in quasi-closed form [6]. On the other hand, the logarithmic singularities of the elliptic integrals involved in the cross-correlations have been handled by means of MaRokhlin-Wandzura quadrature rules [6]. Thanks to the use of all these tricks, the double finite integrals leading to the MoM matrix entries can be evaluated with an accuracy of several significant figures when the functions to be integrated are only evaluated a few hundred times.

\section{NUMERICAL RESULTS}

Both the novel hybrid MoM and the traditional spectral MoM have been applied to the analysis and design of dualpolarization reflectarray antennas made either of stacked rectangular patches [2], [6] or two orthogonal sets of parallel dipoles [3], [4]. The hybrid MoM always outperforms the spectral MoM by a CPU time reduction factor which ranges from 10 to 30 , depending on the antenna. As an example, in Fig. 1(a) and Fig. 1(b), we present the results obtained with both MoM approaches in the analysis of the contoured beam dual-polarization reflectarray antenna with South American coverage that is described in [4]. This antenna consists of 7720 elements arranged in a periodic rectangular grid of period $10 \mathrm{~mm} \times 12 \mathrm{~mm}$. Each element consists of two sets of four parallel dipoles, each set controlling the phase of one of the two polarizations. Note the results obtained in Fig. 1(a) and Fig. 1(b) for the contoured lines of the co-polar gain patterns with both MoM approaches overlap, and pretty good agreement is obtained between the numerical results and the experimental results. For this particular case, the hybrid MoM turns out to be around 20 times faster than the spectral MoM in the analysis of the whole antenna.

\section{CONCLUSIONS}

A novel hybrid spatial-spectral MoM is introduced for the analysis of multilayered periodic structures containing an arbitrary number of stacked patches or sets of orthogonal parallel dipoles in the unit cell. When applied to the analysis and design of reflectarray antennas under the local periodicity assumption, the hybrid MoM turns out to be between 10 and 30 times faster than the traditional pure spectral domain MoM.

\section{ACKNOWLEDGMENT}

This work is supported by "Junta de Andalucía" (project P12-TIC-1435), by European Space Agency under ESTEC

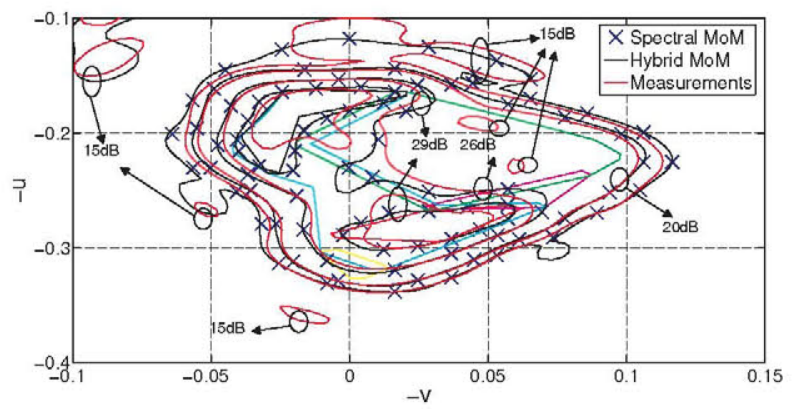

(a)

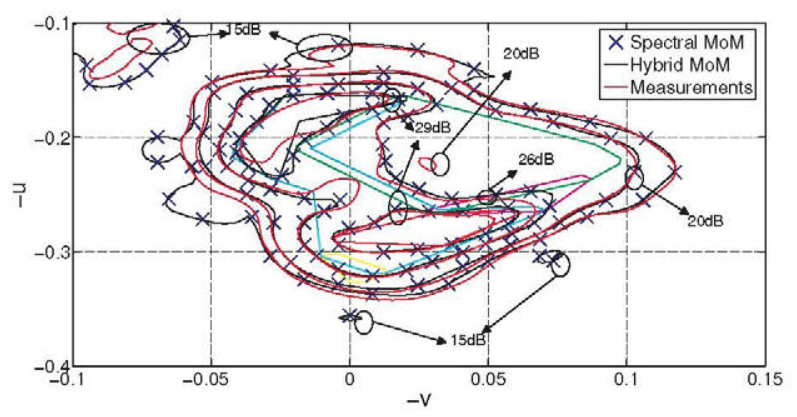

(b)

Fig. 1. Contoured lines for the co-polar gain patterns of the contoured beam dual-polarization reflectarray antenna described in [4] at $11.95 \mathrm{GHz}$ Results are presented for (a) H-polarization and (b) V-polarization. Our results obtained with both the hybrid MoM and the spectral MoM under the local periodicity assumption are compared with measurements. The templates for South American coverage are borrowed from [2] and [3].

Contract No, 4000117113/16/NL/AF, and by the Spanish Ministry of Economy and Competitiveness (projects CICYT TEC2016-75103-C2-1-R and CICYT TEC2013-41913-P).

\section{REFERENCES}

[1] J. Huang and J. A. Encinar, Reflectarray Antennas. NJ: Wiley-IEEE Press, 2008.

[2] J. A. Encinar, M. Arrebola, L. F. de la Fuente, and G. Tosso, "A transmitreceive reflectarray antenna for direct broadcast satellite applications," IEEE Trans. Antennas Propag., vol. 59, pp. 3255-3264, Sep. 2011.

[3] R. Florencio, J. A. Encinar, R. R. Boix, V. Losada, and G. Toso, "Reflectarray antennas for dual polarization and broadband telecom satellite applications," IEEE Trans. Antennas Propag., vol. 63, No. 4 pp. 1234-1246, Apr. 2015.

[4] J. A. Encinar, R. Florencio, M. Arrebola, M. A. Salas, M. Barba, R. R. Boix, and G. Toso "Dual-polarization reflectarray in Ku-band based on two layers of dipole-arrays for a transmit-receive satellite antenna with South American coverage," Proceedings of the 11th European Conference on Antennas and Propagation, EuCAP 2017, pp. 80-83, Paris, France, March 2017.

[5] R. Mittra, C. H. Chan, and T. Cwik, "Techniques for analyzing frequency selective surfaces-a review", Proc. IEEE. vol. 76, no. 12, pp. 1593-1615, Dec. 1988.

[6] R. Florencio, R. R. Boix, and J. A. Encinar, "Fast and accurate MoM analysis of periodic arrays of multilayered stacked rectangular patches with application to the design of reflectarray antennas", IEEE Trans. Antennas Propag., vol. 63, pp. 2558-2571, June 2015.

[7] A. M. Lerer and A. G. Schuschinsky, "Full-wave analysis of threedimensional planar structures," IEEE Trans. Microwave Theory Tech., vol. 41, pp. 2002-2015, Nov. 1993.

[8] T. Fukushima, "Precise and fast computation of elliptic integrals and elliptic functions," 2015 IEEE 22nd Symposium on Computer Arithmetic, pp. 50-57, DOI 10.1109/ARITH.2015.15. 